\title{
Effectiveness and safety of leflunomide for pulmonary and extrapulmonary sarcoidosis
}

\author{
D.H. Sahoo*, D. Bandyopadhyay*, M. Xu”, K. Pearson*, J.G. Parambil*, C.A. Lazar*, \\ J.T. Chapman* and D.A. Culver*,
}

ABSTRACT: Leflunomide has been reported as an alternative therapy in sarcoidosis. However, the published data are limited.

We performed a retrospective chart review of the tolerance and effects of leflunomide therapy in patients with sarcoidosis.

76 patients were included. The most common reasons for initiation were progression of disease or failure of other immunomodulator therapy. Side-effects attributable to leflunomide were noted in $34 \%$ of subjects, prompting discontinuation in $17 \%$. The lungs were a target of therapy in 33 (44\%) and extrapulmonary organs were a target in $45(59 \%)$. The mean \pm SD change in forced vital capacity in the 6 months prior to leflunomide was $-0.1 \pm 0.3 \mathrm{~L}$, and it was $+0.09 \pm 0.3 \mathrm{~L}$ in the following 6 months $(p=0.01)$. For extrapulmonary target organ response, $51 \%$ had a good response and $32 \%$ a partial response. The median corticosteroid dose at initiation was $10 \mathrm{mg}$ (interquartile range 5-20) $\mathrm{mg}$ at baseline, and $0(0-10) \mathrm{mg}$ at the 6-month follow-up $(p<0.001)$.

Leflunomide is a viable alternative agent for pulmonary and extrapulmonary sarcoidosis. Leflunomide appears to facilitate reduction of steroid dose and can be considered as monotherapy or as add-on therapy in cases of progressive disease.

KEYWORDS: Extrapulmonary, leflunomide, pulmonary, sarcoidosis, side-effects, toxicity

$\mathbf{T}$ reatment options for sarcoidosis are expanding rapidly, as medications approved by regulatory agencies for use in other immune-mediated inflammatory diseases have been adopted by the sarcoidosis community. The data supporting the use of several steroid-sparing agents are mainly limited to small case series, and there is a need for further description of clinical experiences with steroid-sparing therapies.

Leflunomide is an oral anti-lymphocyte agent that has been approved by the Food and Drug Administration (FDA) since 1998 for treatment of rheumatoid arthritis. Its putative mechanism of action involves the inhibition of dihydroorotate dehydrogenase, a key enzyme in the de novo synthesis of deoxyuridine monophosphate (dUMP). Since activated, but not memory, T-lymphocytes depend on de novo pyrimidine production for membrane biosynthesis, clonal expansion and terminal differentiation into effector cells, leflunomide represses lymphocyte responses only for actively stimulated lymphocyte clones $[1,2]$. In the absence of sufficient intracellular dUMP, p53mediated apoptosis is triggered in activated, but not resting, lymphocytes [3].

Granulomatous inflammation in sarcoidosis requires antigen-specific CD4+ T-lymphocytes [4, 5].
Inhibition of lymphocyte activation and proliferation is therefore an attractive therapeutic strategy. BAUGHMAN and LOWER [6] previously reported a favourable experience in a single-centre retrospective review of leflunomide for 32 patients with failure of or toxicity from methotrexate. We have been using leflunomide for sarcoidosis since 2004 for pulmonary and extrapulmonary manifestations. We conducted a retrospective chart review to assess the effectiveness of leflunomide in our population as well as to report our experiences with tolerance and toxicity.

\section{MATERIALS AND METHODS}

We identified all sarcoidosis patients for whom leflunomide was prescribed between January 2004 and March 2009 through review of the electronic medical record. All patients met standard criteria for the diagnosis of sarcoidosis [5]. Organ involvement was classified according to criteria proposed in the ACCESS (A Case Control Etiologic Study of Sarcoidosis) formulation [7]. Clinical records were reviewed to determine the target organ(s) precipitating the use of leflunomide. Patients for whom two immunomodulatory drugs (e.g. leflunomide and infliximab) were started at the same time were excluded from the effectiveness analysis, but patients for whom leflunomide was added
AFFILIATIONS

${ }^{*}$ Respiratory Institute,

\# Quantitative Health Sciences, and "Dept of Pathobiology, Cleveland Clinic Foundation, Cleveland, $\mathrm{OH}$, USA.

CORRESPONDENCE

D.A. Culver

Respiratory Institute and Dept of

Pathobiology

Cleveland Clinic

9500 Euclid Avenue

A90

Cleveland

$\mathrm{OH} 44195$

USA

E-mail: culverd@ccf.org

Received:

Dec 162010

Accepted after revision:

April 042011

First published online:

May 122011 
to a stable dose of other medications were included. This study was approved by the Cleveland Clinic Institutional Review Board (Cleveland, OH, USA) under approval number 09-873.

The effects of therapy on extrapulmonary disease were assessed according to the criteria described by BAUGHMAN and LOWER [6]. The therapeutic assessments were obtained from the chart as documented by the treating physician and were re-reviewed by an investigator. Complete response was defined as $>90 \%$ improvement of the maximal disease involvement, partial response required $>50 \%$ reduction in the maximal disease involvement, and patients with $<50 \%$ improvement in disease and/or progressive disease in one or more organs were classified as "no response". The analysis of effectiveness was made at the first visit occurring after 6 months on therapy, but no later than 9 months. Only patients who had follow-up for $\geqslant 6$ months after initiation of leflunomide were included for the effectiveness analysis. For toxicity follow-up, we included all data up to the most recent clinic visit.

The effect of leflunomide on lung function was assessed by comparing the change in lung function over the 6-month period prior to initiation of leflunomide with that occurring at 6 months after initiation using a paired t-test [8]. Pulmonary function testing included for this analysis had to be performed within 12 weeks after the 6-month time-point to be included. All spirometry data were obtained at our centre using the modified spirometry manoeuvre described by STOLLER et al. [9].

A paired t-test or Mann-Whitney U-test was used to test the effects of leflunomide on outcomes, according to the distribution of data. SAS 9.1 software (SAS Institute Inc., Cary, NC, USA) was used for statistical analysis.

\section{RESULTS}

We identified 76 patients with a diagnosis of sarcoidosis who received a prescription for leflunomide. The demographic characteristics are described in table 1: the majority of the patients were females, European-American, and either current or former smokers. $70(92 \%)$ of the patients exhibited lung involvement, mostly commonly Scadding radiographic stage 2 or 3 .

\begin{tabular}{lc}
\hline TABLE 1 Study population & \\
\hline Subjects $\mathbf{n}$ & 76 \\
Age at initiation of drug yrs & $49 \pm 10$ \\
Median duration of sarcoidosis before initiation of & $5.0 \pm 7.8$ \\
$\quad$ treatment yrs & \\
Females \% & 60 \\
Race \% & \\
$\quad$ European-American & 55 \\
$\quad$ African-American & 45 \\
Smokers/ex-smokers \% & 53 \\
CXR stage \% & \\
$\quad$ Stage 0 & 7 \\
$\quad$ Stage 1 & 19 \\
Stage 2 & 35 \\
Stage 3 & 28 \\
\hline
\end{tabular}

Data are presented as mean \pm SD, unless otherwise stated. CXR: chest radiography.
We classified the main reason(s) for starting leflunomide according to the prescribing physician (table 2). The most common single rationale for starting leflunomide was insufficient response to prior therapy. More than one reason could be present. In $13(17 \%)$ of the patients, there was more than one reason for the use of leflunomide. At the time leflunomide was prescribed, $58(76 \%)$ of patients were receiving oral corticosteroids, with a median prednisone dose of $10 \mathrm{mg}$ (interquartile range (IQR) 5-20 mg) daily. 65 (86\%) of the patients had been on other nonsteroid immunomodulators, most commonly methotrexate (58 patients, $77 \%$ ), for a mean \pm SD period of $23 \pm 39$ months.

Our standard practice is to initiate leflunomide at $20 \mathrm{mg}$ daily; only three patients were loaded with higher doses initially (100 mg daily for 3 days). The three patients who were loaded with $100 \mathrm{mg}$ did not experience toxicity. The mean \pm SD duration of leflunomide therapy in our cohort was $16 \pm 13$ months. All but four patients who remained on leflunomide received $20 \mathrm{mg}$ daily, one patient received $30 \mathrm{mg}$ and the remainder $10 \mathrm{mg}$. Of the 76 patients, $54(71 \%)$ remained on leflunomide and had $\geqslant 6$ months of follow-up at our centre. Of the remaining 22 patients, three patients were lost to follow-up after the medication was prescribed, three patients did not start the medication due to insurance difficulties and the remaining 16 patients discontinued the medication within 6 months. The reasons for discontinuation included gastrointestinal intolerance (four patients), other side-effects (10 patients) and patient preference (two patients).

Side-effects were common ( $34 \%$ of subjects), but usually minor; $14(20 \%)$ patients eventually stopped leflunomide due to toxicity or side-effects (table 3). The most common side-effects were diarrhoea $(25 \%)$ and elevated liver enzymes $(7 \%)$. Diarrhoea responded to dose reduction to $10 \mathrm{mg}$ daily in four patients when tried. None of the patients developed persistent hepatic enzyme derangements or evidence of liver failure. Other possible toxicities noted in our cohort included peripheral neuropathy, arthralgia, blurred vision and hair loss (table 3). Eight patients developed symptoms suggestive of lower respiratory tract infection while on leflunomide. The two who were managed at our institution both required hospitalisation and had radiographic and clinical evidence of pneumonia. It was not possible to distinguish whether the remaining six patients had bronchitis or pneumonia with the available records. All eight subjects were treated with

\section{TABLE 2 Reason for initiation of leflunomide}

\section{Reason for initiation}

$\begin{array}{lc}\text { Subjects } \mathbf{n} & 76 \\ \text { Poor response to prior medication } & 60(79) \\ \text { Pulmonary } & 33(44) \\ \text { Extrapulmonary } & 45(59) \\ \text { Toxicity from therapy } & 13(17) \\ \text { Patient preference to taper steroids } & 3(4) \\ \text { >1 of the above reasons } & 13(17)\end{array}$

Data are presented as $n(\%)$, unless otherwise stated. 


\section{TABLE 3 Side-effects of leflunomide}

Side-effect ${ }^{\#}$

$\begin{array}{lc}\text { Total } \mathbf{n} & 68^{\circ} \\ \text { None } & 45(66) \\ \text { Diarrhoea, nausea or bloating } & 17(25) \\ \text { Hepatic enzyme elevation } & 5(7) \\ \text { Neuropathy } & 2(3) \\ \text { Hair loss } & 2(3) \\ \text { Visual disturbance } & 1(1) \\ \text { Arthralgia } & 1(1)\end{array}$

Data are presented as $n(\%)$, unless otherwise stated. \#: the sum is $>100 \%$ since some patients had more than one side-effect; ${ }^{\bullet}$ : eight patients did not take leflunomide after initial prescription.

antibiotic therapy and had resolution of their symptoms, and leflunomide was continued without difficulties in six of them.

Of the 54 patients who completed at least 6 months of treatment and had adequate follow-up, 41 patients were on prednisone at the time leflunomide was started. At 6 months, 13 patients were weaned entirely off systemic corticosteroids. Overall, the median (IQR) prednisone dose at initiation was 10 $(5-20) \mathrm{mg}$ and $0(0-10) \mathrm{mg}$ at 6 months' follow-up $(\mathrm{p}<0.001)$. $36(87 \%)$ out of 41 patients who used any corticosteroids during the study period were able to reduce the dose by $\geqslant 50 \%$, whereas two (5\%) subjects required increased prednisone. Concomitant immunomodulators had been stopped in $16(45 \%)$ out of the 35 patients who were using them prior to starting leflunomide therapy.

The lungs were considered to be a target for initiation of leflunomide in $33(44 \%)$ of patients, of whom 24 completed $\geqslant 6$ months follow-up. We assessed the effect of leflunomide on lung function in this group by comparing the change in forced vital capacity (FVC) in the 6-month period prior to initiation of leflunomide to that occurring at 6 months after initiation (fig. 1). Prior to leflunomide, the mean $\pm \mathrm{SD}$ change in FVC was $-0.1 \pm 0.3 \mathrm{~L}$; after starting the medication, there was a mean gain of $0.09 \pm 0.3 \mathrm{~L}(\mathrm{p}<0.01$, paired $\mathrm{t}$-test). The effect of leflunomide on change in the FVC slope was not dependent on the initial FVC, radiographic stage, disease duration, age, sex or smoking status, but the overall numbers were too small to exclude any relationship between these variables and the outcome. The mean $\pm \mathrm{SD}$ decrease in the diffusing capacity of the lung for carbon monoxide $(D \mathrm{~L}, \mathrm{CO})$ in the 6 months prior to leflunomide was $-0.8 \pm 3.1 \mathrm{~mL} \cdot \mathrm{min}^{-1} \cdot \mathrm{mmHg}^{-1}$; in the 6 months after starting leflunomide, the mean \pm SD gain was $0.6 \pm 2.8 \mathrm{~mL} \cdot \mathrm{min}^{-1} \cdot \mathrm{mmHg}^{-1}$ $(p=0.16$; fig. $1 b)$. Response rates for FVC and $D L, C O$ were nearly identical when the patients were stratified by the reason for starting leflunomide.

Leflunomide was prescribed for treatment of 45 extrapulmonary organ targets in 38 patients; of these, 28 patients with 37 target organs completed $\geqslant 6$ months treatment with leflunomide. The most frequent extrapulmonary manifestations were cutaneous (32\% of the patients assessed for effectiveness), ocular $(21 \%)$ and sinonasal disease $(16 \%)$. No patient had
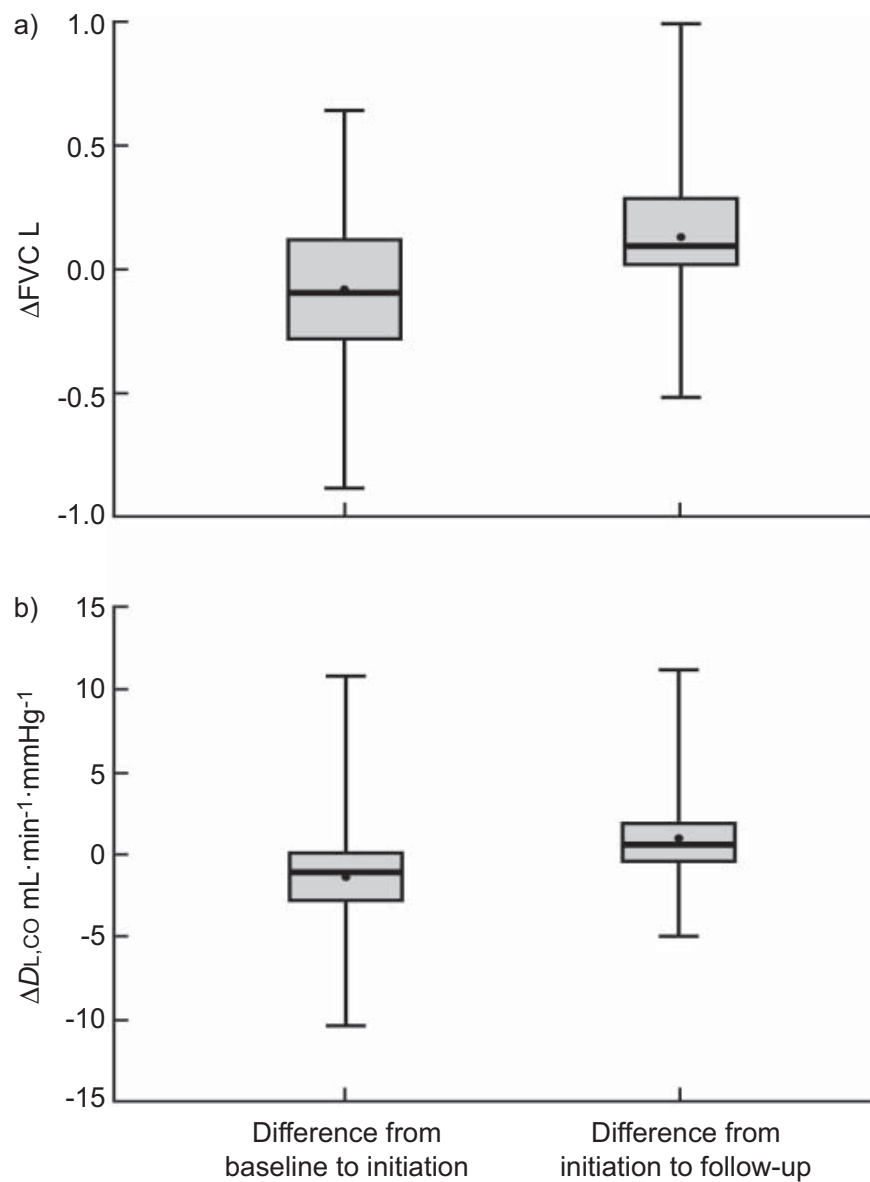

FIGURE 1. Change in $(\Delta)$ a) forced vital capacity (FVC) and b) diffusing capacity of the lung for carbon monoxide $(D\llcorner, \mathrm{CO})$ from initiation of leflunomide to $6-$ 9 months' follow-up. Rectangles represent the interquartile range; the horizontal line within the box is the median and dot denotes mean value. Change in FVC was statistically significant $(p<0.01)$ while $D L, C O$ was not $(p=0.16)$.

concomitant improvement in a target organ and progressive disease in a different organ. Of the 37 target organs, 19 (51\%) had a complete response and $12(32 \%)$ a partial response. There was a trend for a better response in subjects on combination methotrexate/leflunomide therapy than for leflunomide monotherapy $(p=0.004$, Fisher's exact test) (table 4).

\section{DISCUSSION}

The decision to treat sarcoidosis is based on the clinical phenotype of the disease, its perceived effect on organ function and quality of life, and discussions with the patient [10, 11]. There are no FDA-approved drugs for the treatment of sarcoidosis. Most authors recommend corticosteroids as the mainstay of treatment $[5,10,11]$, but there is a growing recognition that the chronic use of corticosteroids may be overly burdensome for some patients due to their toxicities $[12,13]$. Some data suggest that the use of corticosteroids is associated with impaired quality of life, even when taking disease severity into account [14]. In chronic sarcoidosis, it may be worthwhile to have risk-benefit assessment to prioritise steroid-sparing therapies more heavily in clinical decisionmaking, although this hypothesis has not been formally tested. 


\begin{tabular}{|c|c|c|c|c|c|}
\hline & Complete (>90\%) & Partial (50-89\%) & No response $(<49 \%)$ & Unable to assess & Tota \\
\hline Eye & 5 & 2 & 0 & 1 & 8 \\
\hline Sinonasal & 4 & 2 & 0 & 0 & 6 \\
\hline Cardiac & 2 & 1 & 0 & 0 & 3 \\
\hline Gastric & 1 & 0 & 0 & 0 & 1 \\
\hline PNS & 0 & 0 & 1 & 0 & 1 \\
\hline Liver & 0 & 1 & 0 & 0 & 1 \\
\hline Vasculitis & 0 & 1 & 0 & 0 & 1 \\
\hline MSK & 0 & 0 & 1 & 0 & 1 \\
\hline Total $^{\#}$ & 19 & 12 & 5 & 1 & 37 \\
\hline
\end{tabular}

Data are presented as n. CNS: central nervous system; PNS: peripheral nervous system; MSK: musculoskeletal; A: leflunomide; B: leflunomide+methotrexate. ${ }^{\#}$ : Fisher's exact test gave $p=0.004$ between $A$ and $B$.

Unfortunately, once the decision to use steroid-sparing therapies is made, there are few data to guide the clinician. Methotrexate is the most studied alternative therapy, and the choice of most sarcoidosis experts [15]. We use methotrexate in our centre as the preferred second-line option. Other commonly touted nonbiological therapies include azathioprine, leflunomide, mycophenolate and anti-malarial drugs. However, there are no data comparing any of these options and the scientific data supporting their use in sarcoidosis is generally extremely weak [11]. For the past several years, we have routinely preferred leflunomide as the third-line agent after methotrexate (when biological therapies are not indicated) in patients with progressive disease and in those with toxicities from the other medications. This preference has been based on our anecdotal experience with these options.

The first successful use of leflunomide for sarcoidosis was reported in 2003 by MAJITHIA et al. [16] for sinonasal sarcoidosis. Subsequently, BAUGHMAN and LOWER [6] described their experience in 32 subjects, with a partial or complete response present in $78 \%$. Of note, patients intolerant to methotrexate were usually successfully treated with leflunomide in that series. We observed a similar pattern: of the 33 patients who were started on leflunomide because of toxicity from other immunomodulatory medications, 20 tolerated the leflunomide well. Of these, 13 out of 24 patients who switched from methotrexate because of toxicity tolerated leflunomide. Compared with the cohort of BAUGHMAN and LOWER [6], our patient group was younger (mean age 50 versus $40 \mathrm{yrs}$ ) and had a higher frequency of pulmonary disease as a treatment target. The incidence of leflunomide toxicity that precipitated discontinuation was higher in our series (18\% versus $9 \%$ ); this finding may relate to more aggressive dosing in our population: $>90 \%$ of our patients received $20 \mathrm{mg}$ daily, whereas $56 \%$ of the patients in the prior series were treated with $10 \mathrm{mg}$ daily. Other factors that may account for the higher incidence of side-effects in our series include the longer follow-up period, different use of concomitant medications, clinician preferences or other patient-related factors. It is difficult to compare the response rates between the two studies, but overall, they appear to be roughly similar, with complete or good responses noted in $82 \%$ of extrapulmonary organs in our series versus $78 \%$ in the prior experience.

The safety of leflunomide has been well studied in rheumatoid arthritis both as monotherapy and in combination with other immunomodulators [2, 17-19]. In the rheumatoid arthritis population, $50-70 \%$ of patients remained on leflunomide at the end of $1 \mathrm{yr}[18,20]$; the most common reasons for stopping leflunomide in those studies were side-effects (40\%), lack of efficacy (33\%) or both (26\%) [21]. We had a $60 \%$ retention of leflunomide at 12 months in our patients, including $100 \%$ retention in three elderly patients aged $>65$ yrs. A recent prospective, open-label observational series of 334 subjects treated with leflunomide noted that diarrhoea (3.0\%), nausea $(2.4 \%)$, hypertension $(1.8 \%)$ and headache $(1.5 \%)$ were the most common toxicities, with serious adverse drug reactions in four patients $(1.2 \%)$ [22]. The overall incidence of diarrhoea has been reported to be up to $24 \%$ in some studies, but only $2.2 \%$ required discontinuation of the medication [2]. We found that side-effects occurred commonly in our patients (34\%), with a similar incidence of diarrhoea $(25 \%)$ in our population. When it was attempted, four patients responded very well to dose reduction. Our design biases the results toward reporting toxicity, since the effectiveness analysis included only those patients with 6-months' follow-up, but the toxicity could occur at any point during the follow-up period.

The most serious reactions we noted were lower respiratory tract infections and peripheral neuropathy. Review of the eight episodes of lower respiratory tract infection in our population revealed that most of them were treated on an outpatient basis except two, who had severe pneumonia, requiring hospitalisation and withdrawal of immunosuppressive medications. Of these eight patients, six were on leflunomide and prednisone 
and two were on the combination of leflunomide and methotrexate. It is possible that some of our subjects actually developed pneumonitis from the medication, a potential toxicity that has been reported in small series in Japan and New Zealand [18, 23, 24]. However, six out of the eight patients continued on the same immunomodulator including leflunomide with no further evidence of untoward pulmonary events.

New symptoms of length-dependent peripheral neuropathy occurred in two of our subjects, on average 4 months after starting the medication. Peripheral neuropathy has been reported as a complication of leflunomide [25]. Our standard approach when patients complain of neuropathic symptoms includes immediately stopping the medication and active removal with cholestyramine. The neuropathic symptoms persisted in both patients, but partially abated after stopping the medication with no evidence of progression. In both cases, the symptoms remain mild. However, this is a potentially very serious complication if it is not recognised and addressed promptly.

Our study was statistically significant for prednisone dose reduction and FVC change after initiation of treatment with leflunomide. Although the mean change in FVC was only $200 \mathrm{~mL}$, which could be construed as clinically unimportant, the data demonstrate reversal of established declining FVC, which is likely to be relevant in pulmonary sarcoidosis. When comparing the change in FVC between the patients being treated with leflunomide and leflunomide plus methotrexate, in outcome, there was no statistical difference.

There are several weaknesses inherent in our study design. The data are retrospective and uncontrolled. The instrument used for grading disease response is subjective and has not been validated. In support of the grading system, the clinicians caring for the patients were generally able to reduce the dose of concomitant medications. Also, there was no prospective attempt to collect adverse events comprehensively. However, most of the patients we reviewed were followed up closely in our centre and routinely notified us of any new symptoms. Monitoring blood tests are almost exclusively sent to us after initiating new medications. Therefore, it is likely that almost all significant toxicities were included in our sample.

\section{Conclusion}

Leflunomide is a viable alternative immunosuppressive agent in the treatment of sarcoidosis with benefits for both pulmonary and extrapulmonary diseases. We noted that both pulmonary and extrapulmonary sarcoidosis responded favourably to leflunomide, contemporaneous with substantial reductions of steroid and nonsteroid medications. The frequency of sideeffects in this cohort suggests that clinicians prescribing leflunomide should monitor patients closely and consider the use of cholestyramine washout when significant toxicity occurs. Our data demonstrate that leflunomide can be useful both as a steroid-sparing agent and for patients failing other therapies. There is a need for prospective comparative studies to evaluate the relative efficacy of leflunomide monotherapy or combination therapy compared with other alternatives.

How does our study advance the field? The use of steroidsparing medications in sarcoidosis is widely recommended but the choice of which one is based on very few published data. The therapeutic effectiveness of leflunomide and its capacity to facilitate reduction of steroid dose are currently not widely accepted. The experience reported here is the largest description of the clinical effect of a steroid-sparing medication for sarcoidosis and it validates a role for leflunomide as a useful steroid-sparing option.

What are the clinical implications of our study? Our experience demonstrates that leflunomide is a viable option for pulmonary and extrapulmonary sarcoidosis. It is useful for patients who have steroidal side-effects, who have not tolerated methotrexate, or who have suboptimal responses to other medications. Periodic monitoring for side-effects is mandatory.

\section{STATEMENT OF INTEREST}

None declared.

\section{REFERENCES}

1 Rückemann K, Fairbanks LD, Carrey EA, et al. Leflunomide inhibits pyrimidine de novo synthesis in mitogen-stimulated T-lymphocytes from healthy humans. J Biol Chem 1998; 273: 21682-21691.

2 Cohen SB, Iqbal I. Leflunomide. Int J Clin Pract 2003; 57: 115-120.

3 Fox RI, Herrmann ML, Frangou CG, et al. How does leflunomide modulate the immune response in rheumatoid arthritis? BioDrugs 1999; 12: 301-315.

4 Culver DA, Thomassen MJ, Kavuru MS. Pulmonary sarcoidosis: new genetic clues and ongoing treatment controversies. Cleve Clin J Med 2004; 71: 88-92.

5 Joint statement of the American Thoracic Society (ATS), the European Respiratory Society (ERS) and the World Association of Sarcoidosis and Other Granulomatous Disorders (WASOG) adopted by the ATS Board of Directors and by the ERS Executive Committee, February 1999. Am J Respir Crit Care Med 1999; 160: 736-755.

6 Baughman RP, Lower EE. Leflunomide for chronic sarcoidosis. Sarcoidosis Vasc Diffuse Lung Dis 2004; 21: 43-48.

7 Judson MA, Baughman RP, Teirstein AS, et al. Defining organ involvement in sarcoidosis: the ACCESS proposed instrument. ACCESS Research Group. A case control etiologic study of sarcoidosis. Sarcoidosis Vasc Diffuse Lung Dis 1999; 16: 75-86.

8 Swigris JJ, Olson AL, Fischer A, et al. Mycophenolate mofetil is safe, well tolerated, and preserves lung function in patients with connective tissue disease-related interstitial lung disease. Chest 2006; 130: 30-36.

9 Stoller JK, Basheda S, Laskowski D, et al. Trial of standard versus modified expiration to achieve end-of-test spirometry criteria. Am Rev Respir Dis 1993; 148: 275-280.

10 Baughman RP, Costabel U, du Bois RM. Treatment of sarcoidosis. Clin Chest Med 2008; 29: 533-548.

11 Lazar CA, Culver DA. Treatment of sarcoidosis. Semin Respir Crit Care Med 2010; 31: 501-518.

12 Sweiss N, Yeager H. Sarcoidosis requiring systemic steroid: why not a steroid-sparing regimen up-front? Sarcoidosis Vasc Diffuse Lung Dis 2010; 27: 3-4.

13 Baughman RP, Winget DB, Lower EE. Methotrexate is steroid sparing in acute sarcoidosis: results of a double blind, randomized trial. Sarcoidosis Vasc Diffuse Lung Dis 2000; 17: 60-66.

14 Cox CE, Donohue JF, Brown CD, et al. Health-related quality of life of persons with sarcoidosis. Chest 2004; 125: 997-1004.

15 Schutt AC, Bullington WM, Judson MA. Pharmacotherapy for pulmonary sarcoidosis: a Delphi consensus study. Respir Med 2010; 104: 717-723.

16 Majithia V, Sanders S, Harisdangkul V, et al. Successful treatment of sarcoidosis with leflunomide. Rheumatology (Oxford) 2003; 42: 700-702. 
17 Pinto P, Dougados M. Leflunomide in clinical practice. Acta Reumatol Port 2006; 31: 215-224.

18 White DH, Lynskey NV, Jones PB. Leflunomide use in New Zealand. A national prospective post-marketing study. Intern Med J 2009; 39: 95-102.

19 Savage RL. Leflunomide in combination therapy for rheumatoid arthritis. Drug Saf 2010; 33: 523-526.

20 Emery P, Cannon G, Holden W, et al. Results from a cohort of over 40,000 rheumatoid arthritis (RA) patients: adverse event (AE) profiles of leflunomide (LEF), methotrexate (MTX) and other disease-modifying antirheumaticdrugs (DMARDs). Ann Rheum Dis 2002; 61: Suppl. 1, 42.

21 Bettembourg-Brault I, Gossec L, Pham T, et al. Leflunomide in rheumatoid arthritis in daily practice: treatment discontinuation rates in comparison with other DMARDs. Clin Exp Rheumatol 2006; 24: $168-171$.

22 Kellner H, Bornholdt K, Hein G. Leflunomide in the treatment of patients with early rheumatoid arthritis-results of a prospective non-interventional study. Clin Rheumatol 2010; 29: 913-920.

23 Hirabayashi Y, Shimizu H, Kobayashi N, et al. Leflunomideinduced pneumonitis in a patient with rheumatoid arthritis. Intern Med 2006; 45: 689-691.

24 Sawada T, Inokuma S, Sato T. Leflunomide-induced interstitial lung disease: prevalence and risk factors in Japanese patients with rheumatoid arthritis. Rheumatology (Oxford) 2009; 48: 1069-1072.

25 Alcorn N, Saunders S, Madhok R. Benefit-risk assessment of leflunomide: an appraisal of leflunomide in rheumatoid arthritis 10 years after licensing. Drug Saf 2009; 32: 1123-1134. 